\title{
論文
}

GNSS 반송파 위상을 이용한 정밀 측위에서 미지정수 전파기법

한덕희*, 윤희학*, 박찬식**

\section{Integer ambiguity propagation method for a precise positioning using GNSS carrier phase measurements}

\author{
Deokhee Han*, Heehak Yun* and Chansik Park**
}

\begin{abstract}
Many researches on the GNSS integer ambiguity resolution methods for precise positioning and attitude determination applications have been done. However, by the time invariant property of the integer ambiguity, the reuse of integer ambiguity without performing time consuming integer search procedure is possible. In this paper, a new efficient integer ambiguity propagation method is proposed. The initial integer ambiguity can be determined using the famous LAMBDA method and it is propagated with the propagation method. The proposed method can reconfigure the integer ambiguity using the previous epoch's integer ambiguity and new carrier phase measurements under environmental variations such as geometry changes, signal blockage and reacquisition. Experiments with real measurements show the proposed method can determine an integer ambiguity effectively.

\section{초 록}

GNSS 반송파 위상 측정치를 사용하는 정밀 측위 혹은 자세결정에서 미지정수의 결정이 반드시 필요하며 많은 연구가 진행되었다. 그러나 한번 구해진 미지정수는 위성 추적상태 가 변화하지 않으면 상수로 계속 사용할 수 있으므로 미지정수 검색과정을 통하지 않고 이미 구해진 미지정수를 사용할 수 있다. 본 논문에서는 $\mathrm{ARCE}$ 를 변형하여 한번 구해진 미지정수와 새로운 측정치를 이용하여 환경 변화에서도 미지정수를 재구성할 수 있는 미 지정수 전파기법을 제안하였다. 초기 미지정수는 LAMBDA로 구하며 신뢰도 향상을 위하 여 여러 epoch 동안 연속해서 비율검사를 통과한 미지정수를 사용한다. 한번 구해진 미지 정수는 위성의 배치, 위성 신호의 단락 등의 변화에도 미지정수 검색기법을 통하지 않고 제안한 기법을 사용하여 계속 유지될 수 있다. 이로 인하여 미지정수 결정의 성공률이 향 상되며 계산량이 감소되는 효과를 얻을 수 있다. 실제 측정치를 이용한 실험으로 제안한 방법은 효과적인 방법임을 확인하였다.
\end{abstract}

Key Words : LAMBDA, ARCE, Integer Ambiguity Resolution(미지정수 결정), Integer Ambiguity Propagation(미지정수 전파)

† 2009년 3월 9일 접수 2009년 5월 29일 심사완료

* 정회원, 충북대학교 제어로봇공학과 대학원

** 정회원, 충북대학교 제어로봇공학과 교신저자, E-mail : chansp@chungbuk.ac.kr 충북 청주시 흥덕구 성봉로 410 번지

\section{I. 서 론}

GNSS(Global Navigation Satellite System)의 반송파 위상측정치를 이용하면 수 $\mathrm{mm}$ 정도의 정 
밀도로 위치를 구할 수 있다. 그러나 이를 위해 서는 반송파 위상에 포함된 미지정수가 결정되어 야 한다. 미지정수 결정은 해석적인 해가 존재하 지 않아 검색을 통하여 구해야 하므로 검색범위 의 축소 등 계산 량 감소에 대한 많은 연구가 이 루어졌으며 LAMBDA(Least squares AMBiguity Decorrelation Adjustment)[1-3], ARCE(Ambiguity Resolution with Constraint Equation)[4]등이 대 표적인 방법이다. $\mathrm{LAMBDA}$ 는 기본적으로 정수 의 조건을 갖는 정수 최소자승법 (ILS: Integer Least-Squares)의 해를 구하는 방법으로 체계적인 이론과 더불어 뛰어난 성능과 계산상의 이점을 갖고 있어 많은 시스템에서 적용되고 있다. $\mathrm{ARCE}$ 는 3 개의 독립 미지정수만 결정하면 나머 지 종속 미지정수는 검색 없이 직접 계산하여 구 하므로 효율적으로 검색을 수행하는 장점을 가지 므로 자세결정 시스템 등에서 많이 사용된다. GPS(Global Positioning System) 현대화와 Galileo에서 많은 주파수 대역에 신호를 송출할 예정이며 이를 고려한 Wide lane[5], TCAR (Triple Carrier Ambiguity Resolution)[6], CIR (Cascade Integer Resolution)[7]등의 기법이 소개 되고 있지만 이들 기법은 검색 범위 내 검색 후 보의 수를 줄일 수 있는 장점이 있지만 측정잡음 의 영향에 민감하고 반드시 두 개 이상의 주파수 에서 측정치를 얻어야 하므로 저가의 단일주파수 수신기를 이용하는 응용에는 이용할 수 없다. 반 면에 LAMBDA와 ARCE는 GPS의 L1 단일주파 수뿐 아니라 이중주파수, 삼중주파수에도 변경 없이 사용 가능하며, GLONASS(GLObal Navigation Satellite System), Galileo에도 쉽게 적용 가능한 장점이 있다.

현재까지의 미지정수 결정기법은 정수의 제약 조건으로 검색을 통하여 미지정수를 결정하며 이 에 따라 계산 량이 많다. 그러나 미지정수는 시 간에 따라 변화하지 않는 상수이므로 일단 미지 정수를 구하고 나면 매 epoch 측정치에 대하여 미지정수를 구하지 않고 구해진 미지정수를 계속 사용 할 수 있으나 아직 구해진 미지정수를 계속 사용하는 구체적인 방법이 발표되지 않았다. 또 한 GNSS 측정치에서 위성 신호의 단절 및 추가, 사이클 슬립(cycle slip)의 발생에 따라 미지정수 가 바뀌며 이 경우에는 다시 미지정수를 결정해 야 해야 하므로 이를 고려한 미지정수 결정기법 이 필요하다.

본 논문에서는 기존에 잘 알려진 $\mathrm{LAMBDA}$ 와 $\mathrm{ARCE}$ 두 가지 미지정수 결정기법을 이용하여 미 지정수를 효과적으로 결정하고 전파하는 기법을 제안하였다. 초기 측정치를 이용하여 LAMBDA로
미지정수를 결정하고, 이후 측정치에 대해서는 $\mathrm{ARCE}$ 를 기반으로 구성된 미지정수 전파기법으 로 미지정수를 결정한다. 이를 위하여 먼저 $\mathrm{ARCE}$ 를 변형하여 이미 구해진 미지정수와 새로 운 측정치를 이용하여 위성의 배치, 위성 신호의 단락 및 추가 등의 환경 변화에서도 미지정수 검 색 없이 재구성할 수 있는 전파기법을 제안하였 다. 그러나 사이클 슬립 등의 영향으로 너무 많 은 위성에 변화가 생겨 직전 측정치와 현재 측정 치의 공통인 위성수가 4 개 이하인 경우에는 미지 정수의 전파가 불가능하며, 이때는 LAMBDA를 이용하여 미지정수를 다시 결정하도록 하였다. 제안한 방법은 계산량이 많은 미지정수 검색과정 을 생략할 수 있으므로 적은 계산 량으로 미지정 수를 결정하는 효과적인 방법으로 실시간 정밀 측위, 자세결정, 편대비행 등의 응용에 적용될 수 있다.

\section{LAMBDA를 이용한 미지정수 결정}

정밀한 위치를 구하기 위해서는 GNSS 반송파 위상측정치를 이용해야 한다. 기준국과 사용자의 두 수신기에서 측정된 반송파 위상측정치는 이중 차분 기법을 이용하여 처리하며, GPS 반송파 위 상측정치는 수 $m m$ 의 해상도를 가지므로, 두 안 테나 사이의 거리가 멀지 않는 경우 $\mathrm{cm}$ 정확도 로 위치를 구할 수 있음이 알려져 있다[1,4]. 정 밀 측위에서는 선형화된 이중 차분된 코드 $(\rho(k))$ 와 반송파 위상 측정치 $(l(k))$ 를 사용하며 이는 다 음 식으로 나타낼 수 있다[1]. 이 절에서는 매 epoch 동일한 과정이 반복되어야 하므로 편의상 epoch을 나타내는 첨자 $k$ 는 생략하였다.

$$
\begin{gathered}
\rho=H b+v \\
l=H b+\lambda a+\omega
\end{gathered}
$$

여기서 $\rho$ 는 이중 차분된 코드 측정치, $l$ 은 이중 차분된 반송파 위상 측정치, $H$ 는 단일 차분된 시 선각벡터, $b$ 는 WGS-84 좌표계에서 기저선 벡터, $\lambda$ 는 반송파 위상의 파장, $a$ 은 이중 차분된 미지정 수, $v \sim\left(0, Q_{D \Phi}\right)=\left(0, D \cdot Q_{\Psi} \cdot D^{T}\right), \omega \sim N\left(0, Q_{D \Phi}\right)$ $=N\left(0, D \cdot Q_{\Phi} \cdot D^{T}\right)$ 로 백색 Gaussian 측정잡음 을 나타내며, $D$ 는 이중 차분 연산자, $Q_{\Psi}$ 와 $Q_{\Phi}$ 는 차분되지 않은 코드와 반송파 위상측정치의 공분 산을 나타낸다. 식 (1)과 (2)를 합하여 식 (3)과 같이 나타낼 수 있다.

$$
y=B b+A a+e
$$


여기서 $y=\left[\begin{array}{ll}\rho & l\end{array}\right]^{T}, \quad B=\left[\begin{array}{ll}H & H\end{array}\right]^{T}, A=\left[\begin{array}{ll}0 & \lambda I\end{array}\right]^{T}$, $e=\left[\begin{array}{ll}v & \omega\end{array}\right]^{T}$ 이며, 측정잡음 $e$ 공분산 행렬은 $Q_{e}=\left[\begin{array}{cc}Q_{D \Psi} & 0 \\ 0 & Q_{D \Phi}\end{array}\right]$ 로 나타난다.

식(3)에서 미지정수 $a$ 와 기저선 벡터 $b$ 를 동시 에 구해야 하지만 미지정수가 정수라는 조건으로 인하여 해석적인 해가 존재하지 않으며, 검색 범 위내의 가능한 모든 미지정수를 검색하는 방법을 사용한다. 미지정수를 결정하는 많은 기법이 알 려져 있으나, 본 연구에서는 가장 대표적인 $\mathrm{LAMBDA}$ 를 사용하였다. LAMBDA는 정수 최소 자승 기법에 근거한 미지정수 검색기법으로, 상 관되어 있는 공분산 행렬과 미지정수들을 Z-변환 을 이용하여 비 상관화하여 검색 공간을 줄여 효 과적으로 검색할 수 있는 방법이다[2,3]

LAMBDA는 식(3)의 이중 차분된 코드와 반송 파 위상의 GNSS 모델을 이용하여 3단계의 절차 를 거쳐 미지정수를 결정한다. 첫 단계에서 미지 정수가 정수라는 조건을 무시하고 최소자승기법 을 통해 실수 미지수를 결정한다. 그 결과 식 (4) 으로 주어진 추정치와 공분산 행렬을 얻는다.

$$
\left[\begin{array}{c}
\hat{b} \\
\hat{a}
\end{array}\right],\left[\begin{array}{cc}
Q_{\hat{a}} & Q_{\hat{a} \hat{b}} \\
Q_{\hat{b} \hat{a}} & Q_{\hat{b}}
\end{array}\right]
$$

두 번째 단계에서는 구해진 실수해 $\hat{a}$ 을 이용 하여 정수해 $\check{a}$ 를 구한다. $(\hat{a}-a)^{T} Q_{\hat{a}}^{-1}(\hat{a}-a) \leq \chi^{2}$ 을 만족하는 범위내의 모든 미지정수 후보 $a$ 에 대하여 $\Omega=(\hat{a}-a)^{T} Q_{\hat{a}}^{-1}(\hat{a}-a)$ 를 최소로 하는 미 지정수 $\check{a}$ 를 구한다. 이 과정에서 미지정수 공분 산 행렬 $Q_{\hat{a}}$ 가 심하게 상관되어 있어 검색의 효 율이 떨어지므로 LAMBDA에서는 정수의 성질을 유지시키는 Z-변환 행렬 $Z$ 를 이용하며 미지정수 와 공분산 행렬을 다음과 같이 변환한다.

$$
z=Z^{T} a, \hat{z}=Z^{T} \hat{a}, Q_{\hat{z}}=Z^{T} Q_{\hat{a}} Z
$$

이를 이용하면 $\Omega=(\hat{z}-z)^{T} Q_{\hat{z}}^{-1}(\hat{z}-z)$ 를 최소로 하는 미지정수 $z$ 를 구하는 문제로 변환되며, 이 때 공분산 행렬 $Q_{\hat{z}}$ 가 대각행렬에 가까운 형태로 변경되어 검색의 효율을 극대화 할 수 있다. 이 경우 미지정수 검색문제는 $\Omega=\sum_{i=1}^{m} \frac{\left(\hat{z}_{i \mid I}-z_{i}\right)^{2}}{\sigma_{i \mid I}^{2}}$ 를 최소로 하는 미지정수 $z$ 를 구하는 문제가 된다. 여기서 $m$ 은 미지정수의 개수, $\sigma_{i \mid I}^{2}$ 는 $Q_{\hat{z}}$ 의 대각 항을 나타낸다. 따라서 미지정수를 순차적으로 구할 수 있으며, 차례로 검색범위가 줄어들어 효
율적으로 검색할 수 있다. 검색범위 내의 모든 후보에 대하여 최소의 $\Omega$ 를 만족하는 $\tilde{z}$ 를 최종 값으로 결정하고 식(5)의 관계를 이용하여 미지 정수 $\check{a}$ 을 구할 수 있다.

세 번째 단계에서는 구해진 미지정수 $\check{a}$ 을 이 용하여 식(6)와 같이 보상된 기저선 벡터 $\check{b}$ 를 구 한다. 이때 공분산 행렬은 $Q_{\tilde{b}}=Q_{\hat{b}}-Q_{\hat{b} \hat{a}} Q_{\hat{a}}^{-1} Q_{\hat{a} \hat{b}}$ 이 된다.

$$
\check{b}=\hat{b}-Q_{\hat{b} \hat{a}} Q_{\hat{a}}^{-1}(\hat{a}-\check{a})
$$

참 미지정수를 찾을 확률은 성공률로 불리며, 이 값은 공분산 행렬 $Q_{\hat{a}}$ 만으로 계산된다. 계산 에 실제 측정치 등 다른 정보를 필요로 하지 않 으므로 성공률은 예측 치를 제공할 수 있다. 성 공률의 최소값은 다음 식으로 계산할 수 있다. 여기서 $\Phi(x)=\int_{-\infty}^{x} \frac{1}{\sqrt{2 \pi}} \exp \left(-\frac{1}{2} z^{2}\right) d z$ 상보오차 함수(erfc: complementary error function)이며 구 해진 미지정수가 참 값임을 보장하기 위해서 성 공률은 1 에 근접한 충분히 큰 값을 가져야 한다.

$$
P(\check{z}=z) \geq \prod_{i=1}^{n}\left(2 \Phi\left(\frac{1}{2 \sigma_{\hat{z}_{i \mid l}}}\right)-1\right)
$$

\section{III. 미지정수 전파기법}

한번 구해진 미지정수는 시간에 따라 변화하 지 않으므로 계속 사용할 수 있다. 그러나 추적 되는 위성의 상태에 변화가 생기는 경우 이를 반 영하여 다시 결정해야 한다. 본 논문에서는 한번 구해진 미지정수를 매번 다시 구하지 않고 계속 사용할 수 있는 $\mathrm{ARCE}$ 에 기반한 미지정수 전파 기법을 제안한다. 제시한 미지정수 전파기법은 GPS 단일주파수 수신기를 기준으로 설명하였지 만 이중주파수와 삼중주파수 수신기에서도 변경 없이 적용할 수 있으며, Galileo와 GLONASS에 서도 사용될 수 있다[4,5].

식 (2)로 주어지는 이중 차분된 반송파 위상측 정치에서, 의 영 공간(null space) 행렬을 다음 과 같이 정의한다.

$$
E=\operatorname{null}\left(H^{T}\right)
$$

$E^{T}$ 를 식 (2)의 양변에 곱하면 식(9)를 얻을 수 있으며, 이를 제한 조건식(Constraint Equation) 으로 정의한다. 여기서 $l_{E} \equiv E^{T} l, \omega_{E} \equiv E^{T} \omega$, $\omega_{E} \sim N\left(0, E^{T} Q_{D \phi} E\right) \equiv N\left(0, Q_{E}\right)$ 이다. 


$$
l_{E}=\lambda E^{T} a+\omega_{E}
$$

모든 미지정수는 매 epoch마다 구해지는 제한 조건식을 위치에 무관하게 만족하여야 한다. 따 라서 이를 이용하면 위치를 구하지 않고서도 미 지정수 검색이 가능하다. 즉 식 (9)의 제한 조건 식에서 미지정수 후보는 다음과 같이 독립 미지 정수 항 $\left(a_{I} \in Z^{3 \times 1}\right)$ 과 종속 미지정수 항 $\left(a_{D} \in Z^{(m-3) \times 1}\right)$ 으로 나눌 수 있다.

$$
a_{C E}=\left[\begin{array}{ll}
a_{I} & a_{D}
\end{array}\right]^{T}
$$

한 번 결정된 미지정수는 독립 미지정수 항에 변화가 없다면, 새로운 측정치에 대하여 매번 종 속 미지정수 항을 직접 결정할 수 있다. 즉 고정 된 독립 미지정수와 새로운 반송파 위상 측정치 로부터 종속 미지정수 항을 다음의 식으로 직접 구할 수 있다. 여기서 $E_{I}$ 는 $a_{I}$ 의 정의에 따라 나 누어진 $E$ 의 부분, $E_{D}$ 는 $a_{D}$ 의 정의에 따라 나누 어진 부분이다.

$$
a_{D}=\text { round }\left(\frac{E_{D}^{-T}}{\lambda}\left(l_{E}-E_{I}^{T} \lambda a_{I}\right)\right)
$$

식(11)을 기반으로 구현한 미지정수 전파기법 을 그림 1 에 나타내었다. 초기 독립 미지정수 값 $a_{I}$ 는 $\mathrm{LAMBDA}$ 에서 구해진 값을 이용한다. 이때 수 epoch에 걸쳐 같은 값으로 구해져 충분히 참 미지정수로 인정할 수 있는 값을 사용한다. 이 상태에서 새로운 반송파 위상 측정치를 수집하고

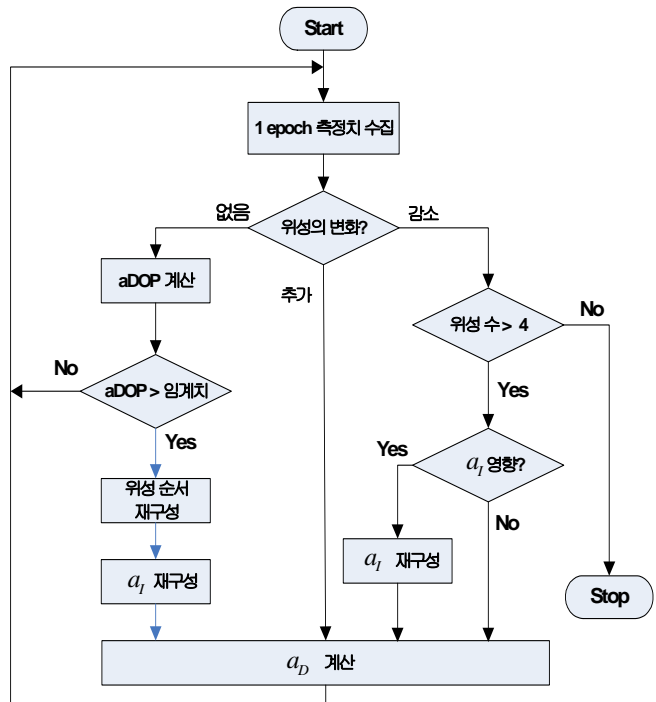

그림 1. 미지정수 전파 과정
위성의 변화 여부를 확인한다. 위성의 추가나 삭 제가 없는 경우에도 미지정수 결정의 성능 유지 를 위하여 식(12)의 $\mathrm{aDOP}($ Ambiguity Dilution of Precision)을 구하고 이 값이 임계치를 벗어나 지 않으며 현재의 상태를 유지한다. 임계치를 벗 어나면 위성의 순서를 재배치하고 이에 따라 독 립 미지정수를 재구성한다. 위성의 순서를 정하 기 위해서는 보이는 위성에 대하여 가능한 순서 를 정하고 이를 이용하여 계산한 $\mathrm{aDOP}$ 중 최소 값을 주는 위성의 순서를 이용한다. 그러나 $m$ 개 의 미지정수에 대하여 $m$ ! 개의 조합에 대한 $\mathrm{aDOP}$ 계산을 해야 하므로 본 논문에서는 앙각 이 높은 위성 6 개에 대해서만 $\mathrm{aDOP}$ 으로 순서를 정하고 나머지는 앙각이 큰 순서로 정하여 계산 량을 감소시켰다. 위성의 순서가 정해지면 이에 따라 독립 미지정수를 재배치한다.

$$
a D O P=\operatorname{trace}\left[E_{D} Q_{E}^{-1} E_{D}^{T}\right]^{-1}
$$

이중 차분된 미지정수와 수신기간 단일 차분 된 미지정수와의 관계를 이용하여 미지정수를 재 구성한다. 식 (13)에 2번째와 3번째 위성의 순서 가 바뀐 경우 새로운 미지정수와 이전 미지정수 와의 관계를 나타내었으며 이로부터 미지정수를 구할 수 있다. 여기서 $a^{i}$ 위성 $i$ 에 대하여 수신기 간 단일 차분된 미지정수를 $a^{i j}=a^{i}-a^{j}$ 는 위성 $i$ 와 $j$ 사이의 이중 차분된 미지정수를 나타내며, 초기 독립 미지정수 $\left[\begin{array}{lll}a^{12} & a^{23} & a^{34}\end{array}\right]^{T}$ 가 새로운 독 립 미지정수 $\left[\begin{array}{ccc}a^{12} & \tilde{a}^{23} & \tilde{a}^{34}\end{array}\right]^{T}$ 로 변경된다[6]. 이 방법을 독립 미지정수 항의 위성이 제거되는 경 우에도 확장할 수 있다. 식 (14)에 3번 위성이 제 거되어 새로운 독립 미지정수를 결정하는 과정의 예를 나타내었다.

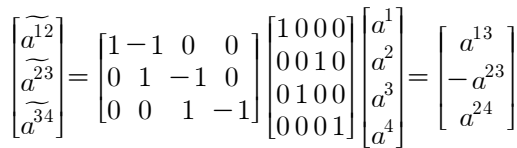

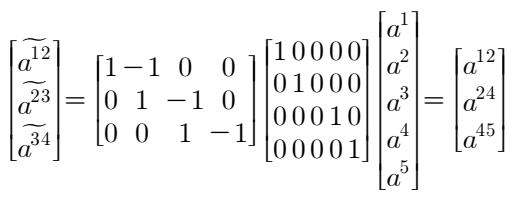

위성이 추가된 경우에는 추가된 위성을 종속 미지정수 항에 포함시켜 식 (11)로 새로운 $a_{D}$ 를 계산한다. 위성이 제거된 경우, 제거된 위성이 독 립 미지정수 항에 영향을 미치면 위성 순서를 재 구성하여 식(14)로 독립 미지정수를 계산하고, 독 립 미지정수 항에 영향을 미치지 않으면 식 (11) 
로 종속 미지정수를 구한다.

반송파 위상 측정치를 이용하기 위해서는 최 소 4 개 이상의 위성이 필요하므로 관측되는 위성 의 수가 4 개미만이 되면 더 이상 진행하지 않는 다. 이후에 다시 위성 수가 4 개 이상이 되면 처 음부터 다시 시작한다.

사이클 슬립은 반송파 위상측정치에 포함된 미지정수 값이 순간적으로 다른 값으로 변화하는 현상으로 미지정수 전파기법에서는 사이클 슬립 을 인식하지 못하면 잘못된 미지정수와 위치를 구하게 되므로 이에 대한 대비가 필요하다. 특히 미지정수 전파기법은 전체 미지정수가 아닌 독립 미지정수 항만을 전파하고 나머지 종속 미지정수 는 이를 이용하여 계산하므로 종속 미지정수에 발생한 사이클 슬립은 영향을 주지 않지만 독립 미지정수에 발생한 사이클 슬립은 치명적인 결과 를 유발할 수 있다. 사이클 슬립의 검출 및 보정 의 대표적인 기법은 $[8,9]$ 에 소개되어 있으며 이 를 이용하면 효과적으로 제거할 수 있다.

\section{IV. 실험 및 성능평가}

제안한 미지정수 결정기법의 성능을 확인하기 위한 실험을 수행하였다. 실험을 위해 2 대의 NovAtel사의 ProPak-V3[10] GPS 수신기를 이용 하여 2009년 2월 16일 22시부터 1시간 동안 $1 \mathrm{~Hz}$ 주기로 측정치를 수집하였다. 기준점과 사용자 사이의 기저선 길이는 $13.96 \mathrm{~m}$ 이고, 차분되지 않 은 코드 측정치와 반송파 위상 측정오차는 평균 0 이고 표준편차가 각각 $20 \mathrm{~cm}, 2 \mathrm{~mm}$ 인 백색잡음 으로 가정하였다.

그림 2-(a)에 실험 동안 관측된 위성 개수와 PDOP(Position Dilution of Precision)을 나타내 었으며, 두 수신기에서 동시에 8 개에서 11 개의 위성이 관측되고, 대부분의 구간에서 $\mathrm{PDOP}$ 은 2

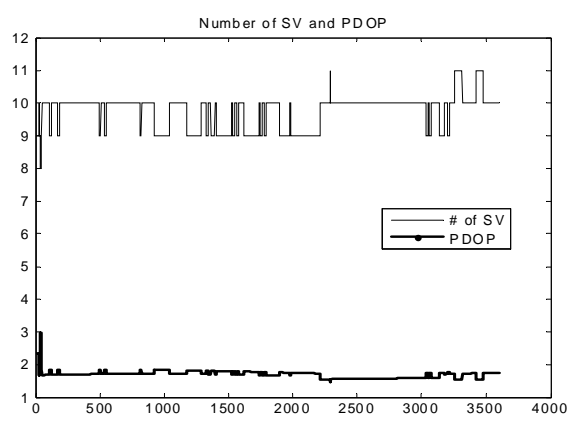

그림 2. 가시위성 수와 PDOP

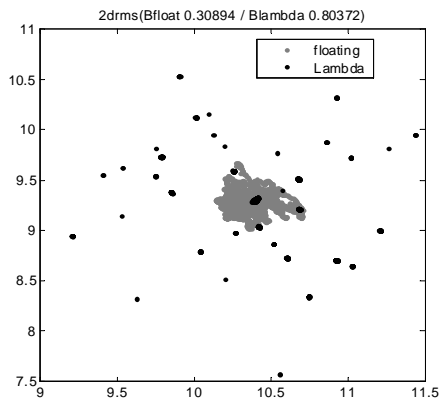

그림 3. 수평면에서의 궤적

(실수해와 LAMBDA 기법으로 구한 위치)

이하를 유지하였다. 앙각이 낮은 PRN 27과 28번 위성의 추적이 단속적으로 이루어져 위성 수의 변화가 심하게 나타났다.

식 (7)을 이용하여 계산된 이론 성공률과 $\mathrm{LAMBDA}$ 를 적용하여 구한 실제 성공률의 평균 값은 각각 $99.97 \%$ 와 $68.14 \%$ 로 나타났다. LAMBDA는 매 epoch 적용하여 미지정수를 구 했으며, 전체측정치를 이용하여 후처리로 구한 참 미지정수와 비교하여 성공률을 계산하였다. 코드와 반송파 위상측정치의 오차와 위성의 배치 로 결정되는 이론 성공률은 거의 1 에 가깝게 나 타나지만 실험값은 다중경로 등의 오차의 영향으 로 실제 성공률이 낮게 나타난 것으로 판단된다.

그림 3에 최종적으로 구해진 위치를 나타내었 다. 그림에서 LAMBDA가 미지정수를 구하지 못 하므로 구해진 미지정수를 이용하여 구한 위치가 넓게 분포됨을 볼 수 있으며 수평면에서의 오차 가 $80 \mathrm{~cm}$ (2dRMS)로 실수해를 사용하여 구한 $30 \mathrm{~cm}$ (2dRMS) 보다 나쁜 결과를 보인다.

그림 4에 미지정수 전파기법을 이용하여 구해 진 3개의 독립 미지정수를 나타내었다. 후 처리 로 구해진 참 미지정수와 비교하여 $100 \%$ 의 정확 도로 미지정수를 구할 수 있었으며, 4-(a)에서 3205 epoch 까지는 PRN 15, 24, 18, 20이 독립미 지정수 결정에 사용되었으나 3206 epoch에서 PRN 10번 위성이 추적되지 않음에 따라 독립미 지정수가 PRN 9, 22, 24, 26으로 변경되어도 동 작함을 볼 수 있다. 4-(b)에 LAMBDA로 구한 미 지정수와 전파기법으로 구한 미지정수의 차를 자 세히 나타내었다.

그림 5에 미지정수 전파기법으로 구해진 위치 를 나타내었다. 수평오차가 $2.8 \mathrm{~cm}(2 \mathrm{dRMS})$ 로 실 수 해를 사용하는 경우나, 매번 LAMBDA를 사 용하는 경우에 비하여 월등히 향상된 결과를 보 

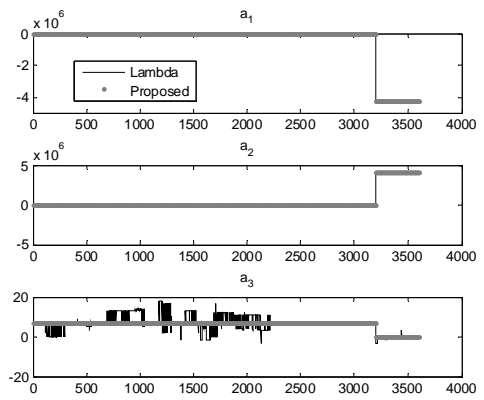

(a) 구해진 독립 미지정수
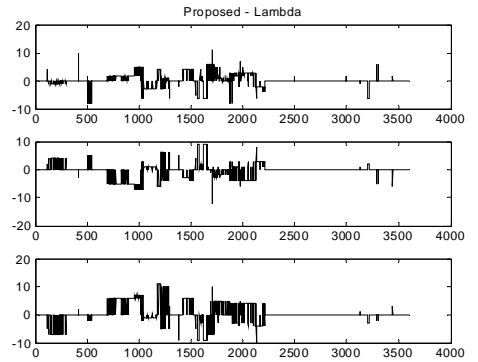

(b) 두 방법의 차

그림 4. 구해진 미지정수 $(3205$ epoch까지 PRN 15-24-18-20, 3206 epoch 이후 PRN 9-22-24-26)

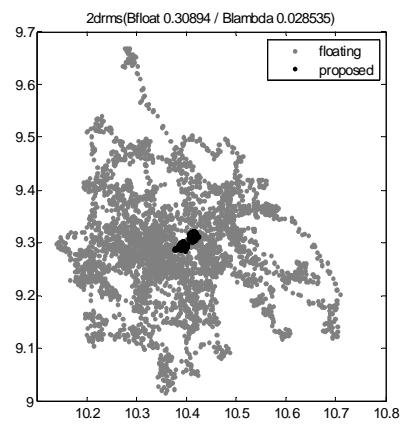

(a) 실수해와 전파기법으로 구한 위치

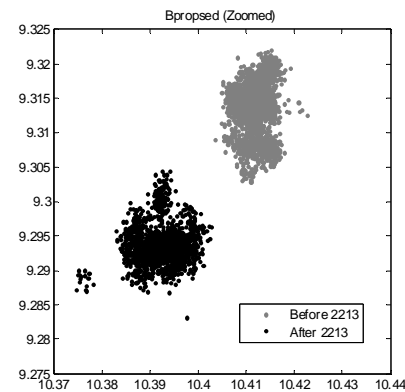

(b) 전파기법으로 구한 위치의 확대

그림 5. 수평면에서의 궤적
여준다. 그림 5-(b)에서 구해진 위치가 2213 epoch에서 PRN 12번이 추가됨에 따라 궤적이 $2 \mathrm{~cm}$ 가량 이동하며 나머지 부분에서도 위성의 추가나 단절에 따라 위치가 조금씩 이동됨을 볼 수 있다. 이는 위성 별 오차의 차이를 고려하지 않고 해를 구하므로 나타나는 현상이며 위성의 앙각을 가중치에 반영하는 가중 최소자승법이나 Kalman 필터에서는 나타나지 않을 것으로 예측 된다.

\section{V. 결 론}

본 논문에서는 GNSS를 이용하는 정밀 항법이 나 자세결정에서 요구되는 미지정수 결정 문제를 효율적으로 처리할 수 있는 미지정수 전파기법을 제안하고 실제 측정치를 이용하여 그 타당성을 확인하였다. 미지정수 전파기법은 처음 몇 epoch 의 측정치와 $\mathrm{LAMBDA}$ 로 구해진 미지정수를 그 이후에는 검색을 통하지 않고 전파하는 방법으로 $\mathrm{ARCE}$ 를 기반으로 설계되었다. 미지정수의 전파 기법에서는 미지정수를 구하면 그 값을 계속 유 지하게 되므로 효과적으로 사용할 수 있다. 실제 측정치를 이용한 실험결과 매 epoch 측정치에 대하여 $\mathrm{LAMBDA}$ 를 이용하면 $68 \%$ 정도의 검색 성공률을 보이는데 비하여 미지정수 전파기법은 $100 \%$ 의 검색 성공률을 보임을 확인하였다.

제안한 방법 단독으로는 미지정수 결정에 사 용될 수 없지만 기존의 LAMBDA 혹은 ARCE와 연계하여 계산량을 줄이고 성공률을 향상시키는 효과적인 방법으로 사용될 수 있다. 특히 기존의 방법의 결과와 전파기법의 결과를 비교하면 구해 진 미지정수의 검증을 보다 효과적으로 수행할 수 있을 것으로 기대된다.

\section{후 기}

이 논문은 2008년도 충북대학교 학술연구지원 사업의 연구비지원에 의하여 연구되었음.

\section{참고문헌}

1) Teunissen P.J.G., A. Kleusberg, GPS for Geodesy, Springer-Verlag, Berlin, 1998.

2)Tenuissen P.J.G., "A new method for fast carrier phase ambiguity estimation", Proceedings of IEEE Position, Location and Navigation Symposium, Lasvegas, Nevada, 1994, pp. 562 573. 
3)de Jonge P.J., and C.C.J.M Tiberius, The LAMBDA Method for Integer Ambiguity Estimation: Implementation Aspects LGR Series No.12, Delft Geodetic Computing Centre, 1996.

4)박찬식, 이장규, 지규인, 이영재, “GPS 반송 파 위상을 이용한 정밀 자세 측정”, 제어자동화 시스템공학 논문지, 제3권 제6호, 1997, pp. 602 512.

5)손석보, 박찬식, 이상정, “L2 측정치를 확장 된 잉여측정치로 이용하는 미지정수 결정기법”, 항공우주학회 논문지, 제 28권 5호, 2000.

6)Teunissen P.J.G., P. Joosten and C. Tiberius, "A Comparison of TCAR, CIR and LAMBDA GNSS Ambiguity Resolution", Proceedings of ION GPS 2002, Portland, Oregon, 2002, pp. 2799 2808.
7)Jung J., P. Enge and B. Pervan "Optimization of Cascade Integer Resolution with three civil GPS frequencies", Proceedings of ION GPS-2000, Salt Lake City,UT, 2000, pp. 2191 2200.

8)de Jong C.D., "Real-time integrity monitoring, ambiguity resolution and kinematic positioning with GPS", Proceedings of 2nd European Symposium GNSS'98, Toulouse, 1998, pp.VIII07/1 VIII07/7.

9)Borre, K., "Receiver clock reset, cycle slip detection, and Receiver autonomous integrity monitoring", Galileo Network, Aalborg University, Nov. 17, 2006.

10)http://www.novatel.com/Documents/Pape rs/ProPakV3.pdf 